\title{
Analysis approach to finite monoids
}

\author{
A Sinan Çevik ${ }^{1 *}$, I Naci Cangül ${ }^{2}$ and Yılmaz Şimşek ${ }^{3}$
}

${ }^{\text {"Correspondence: }}$

sinan.cevik@selcuk.edu.tr

${ }^{1}$ Department of Mathematics,

Faculty of Science, Selçuk University,

Campus, Konya, 42075, Turkey

Full list of author information is

available at the end of the article

\begin{abstract}
In a previous paper by the authors, a new approach between algebra and analysis has been recently developed. In detail, it has been generally described how one can express some algebraic properties in terms of special generating functions. To continue the study of this approach, in here, we state and prove that the presentation which has the minimal number of generators of the split extension of two finite monogenic monoids has different sets of generating functions (such that the number of these functions is equal to the number of generators) that represent the exponent sums of the generating pictures of this presentation. This study can be thought of as a mixture of pure analysis, topology and geometry within the purposes of this journal.
\end{abstract}

AMS Subject Classification: 11B68; 11S40; 12D10; 20M05; 20M50; 26C05; 26C10

Keywords: efficiency; $p$-Cockcroft property; split extension; generating functions; Stirling numbers; array polynomials

\section{Introduction and preliminaries}

Associated with any (connected) topological space $X$ is its fundamental group $\pi_{1}(X)$ or 2-complex (Squier complex) $\mathcal{D}(X)$. This can often be specified by means of a presentation. A presentation of a group $G$ or monoid $M$ consists of a set of generators of $G$ or $M$, together with a collection of relations amongst these generators, such that any other relation amongst the generators is derivable (in a precise sense) from the given relations. Algebraic information about $\pi_{1}(X)$ or $\mathcal{D}(X)$ can be used to obtain topological information about $X(c f$. [1]). Many techniques of this branch of mathematics are purely algebraic, and it is possible to achieve much using these techniques. However, in recent years many techniques involving geometric ideas have emerged and are proving more fruitful. These geometric techniques involve graph theory, the theory of tessellations of various surfaces and covering space theory, to name a few.

The number of vertex-colorings of a graph is given by a polynomial on the number of used colors (see [2]). Based on this polynomial, one can define the chromatic number as the minimum number of colors such that the chromatic polynomial is positive. Recently, our attention has been drawn to the paper [3] which is a generalization on the chromatic polynomial of a graph subdivision, and basically the authors determine the chromatic number for a simple graph and then present the generalized polynomial for a particular case of graph subdivision. In this reference, the main idea was to express some graph theoretical parameters in terms of special functions. In a similar manner within algebra, by considering a group or a monoid presentation $\mathcal{P}$, an approximation from algebra to analysis has been recently developed [4]. To do that, the authors supposed $\mathcal{P}$ satisfies the special algebraic properties either efficiency or inefficiency while it is minimal. (The reason for

(C) 2013 C Cevik et al.; licensee Springer. This is an Open Access article distributed under the terms of the Creative Commons Attribution License (http://creativecommons.org/licenses/by/2.0), which permits unrestricted use, distribution, and reproduction in any medium, provided the original work is properly cited. 
choosing efficiency or (minimal) inefficiency was to have an advantage to work on a minimal number of generators.) Then it was investigated whether some generating functions can be applied, and then it was studied what kind of new properties can be obtained by considering special generating functions over $\mathcal{P}$. In fact, to investigate this theory, $\mathcal{P}$ has been taken as the presentation of the split extension $\mathbb{Z}_{n} \rtimes \mathbb{Z}$ and $\mathbb{Z}^{2} \rtimes \mathbb{Z}$, respectively. Since the results in [3] imply a new studying area for graphs in the meaning of representation of parameters by generating functions, the results in [4] will be also given an opportunity to make a new classification of infinite groups and monoids by using generating functions.

This paper can be thought of as another version of [4]. Our general aim here is to define some generating functions in terms of the minimality of the given presentation. This will imply that the minimal number of generators can be represented as generating functions. Similarly as in [4], our approximation will be applied by considering the split extension. Here, the split extension will be defined as a semi-direct product of two finite monogenic (cyclic) monoids (we may refer to [5] for details on these monoids). It is obvious that the split extension of two finite structures will also be finite. So, the main difference between the results in here and in the paper [4] lies in this fact. Because, while a classification over special cases of infinite groups or monoids was given in [4], the classification in the present paper only focuses on the finite monoids. It is well known that giving some different approximations over finite cases is also as important as giving those over infinite cases.

In the following first subsection, as supportive material, some algebraic facts over split extensions (equivalently, semi-direct products), presentations of finite monogenic monoids, a trivializer set of these presentations and efficiency (equivalently, $p$-Cockcroft property) are reminded. In Section 2, we present the main material of this paper as two separate subsections. In the first subsection, we present some known results about necessary conditions for the presentation, say $\mathcal{P}_{M}$, of the split extension of two finite monogenic monoids to be $p$-Cockcroft (see Proposition 2.1 below) and to be minimal but inefficient (see Proposition 2.3 below). In the final subsection, as a result of all theories until there, we introduce generating functions related to our title (see Theorems 2.5, 2.7 and 2.12 below). In Section 3, by considering one of the functions defined in the previous section, we study this function in the meaning of again generating functions and functional equations (see Theorems 3.1 and 3.3 below).

\subsection{Fundamentals of the algebraic part}

This subsection should be completely thought of as a part of the expressions in the beginning of this paper.

Let $\mathcal{P}=[X ; \mathbf{r}]$ be a monoid presentation where a typical element $R \in \mathbf{r}$ has the form $R_{+}=R_{-}$. Here $R_{+}, R_{-}$are words on $X$ (that is, elements of the free monoid $F(X)$ on $X$ ). The monoid defined by $[X ; \mathbf{r}]$ is the quotient of $F(X)$ by the smallest congruence generated by $\mathbf{r}$.

We have a (Squier) graph $\Gamma=\Gamma(X ; \mathbf{r})$ associated with $[X ; \mathbf{r}]$, where the vertices are the elements of $F(X)$ and the edges are the 4-tuples $e=(U, R, \varepsilon, V)$, where $U, V \in F(X), R \in \mathbf{r}$ and $\varepsilon= \pm 1$. The initial, terminal and inversion functions for an edge $e$ as given above are defined by $\iota(e)=U R_{\varepsilon} V, \tau(e)=U R_{-\varepsilon} V$ and $e^{-1}=(U, R,-\varepsilon, V)$.

Two paths $\pi$ and $\pi^{\prime}$ in a 2-complex are equivalent if there is a finite sequence of paths $\pi=\pi_{0}, \pi_{1}, \ldots, \pi_{m}=\pi^{\prime}$, where for $1 \leq i \leq m$, the path $\pi_{i}$ is obtained from $\pi_{i-1}$ either by inserting or deleting a pair $e e^{-1}$ of inverse edges or else by inserting or deleting a defining path for one of the 2-cells of the complex. There is an equivalence relation, $\sim$, on paths in 
$\Gamma$ which is generated by $\left(e_{1} \cdot \iota\left(e_{2}\right)\right)\left(\tau\left(e_{1}\right) \cdot e_{2}\right) \sim\left(\iota\left(e_{1}\right) \cdot e_{2}\right)\left(e_{1} \cdot \tau\left(e_{2}\right)\right)$ for any edges $e_{1}$ and $e_{2}$ of $\Gamma$. This corresponds to requiring the closed paths $\left(e_{1} \cdot \iota\left(e_{2}\right)\right)\left(\tau\left(e_{1}\right) \cdot e_{2}\right)\left(e_{1}^{-1} \cdot \tau\left(e_{2}\right)\right)\left(\iota\left(e_{1}\right) \cdot e_{2}^{-1}\right)$ at the vertex $\iota\left(e_{1}\right) \iota\left(e_{2}\right)$ to be the defining paths for the 2-cells of a 2 -complex having $\Gamma$ as its 1 -skeleton. This 2-complex is called the Squier complex of $\mathcal{P}$ and denoted by $\mathcal{D}(\mathcal{P})$ (see, for example, [6-9]). The paths in $\mathcal{D}(\mathcal{P})$ can be represented by geometric configurations, called monoid pictures. We assume here that the reader is familiar with monoid pictures (see [6, Section 4], [7, Section 1] or [8, Section 2]). Typically, we will use blackboard bold, e.g., $\mathbb{A}, \mathbb{B}, \mathbb{C}, \mathbb{P}$, as notation for monoid pictures. Atomic monoid pictures are pictures which correspond to paths of length 1 . Write $[|U, R, \varepsilon, V|]$ for the atomic picture which corresponds to the edge $(U, R, \varepsilon, V)$ of the Squier complex. Whenever we can concatenate two paths $\pi$ and $\pi^{\prime}$ in $\Gamma$ to form the path $\pi \pi^{\prime}$, then we can concatenate the corresponding monoid pictures $\mathbb{P}$ and $\mathbb{P}^{\prime}$ to form a monoid picture $\mathbb{P P}^{\prime}$ corresponding to $\pi \pi^{\prime}$. The equivalence of paths in the Squier complex corresponds to an equivalence of monoid pictures. That is, two monoid pictures $\mathbb{P}$ and $\mathbb{P}^{\prime}$ are equivalent if there is a finite sequence of monoid pictures $\mathbb{P}=\mathbb{P}_{0}, \mathbb{P}_{1}, \ldots, \mathbb{P}_{m}=\mathbb{P}^{\prime}$ where, for $1 \leq i \leq m$, the monoid picture $\mathbb{P}_{i}$ is obtained from the picture $\mathbb{P}_{i-1}$ either by inserting or deleting a subpicture $\mathbb{A}^{-1}$, where $\mathbb{A}$ is an atomic monoid picture, or else by replacing a subpicture $(\mathbb{A} \cdot \iota(\mathbb{B}))(\tau(\mathbb{A}) \cdot \mathbb{B})$ by $(\iota(\mathbb{A}) \cdot \mathbb{B})(\mathbb{A} \cdot \tau(\mathbb{B}))$ or vice versa, where $\mathbb{A}$ and $\mathbb{B}$ are atomic monoid pictures.

A monoid picture is called a spherical monoid picture when the corresponding path in the Squier complex is a closed path. Suppose $\mathbf{Y}$ is a collection of spherical monoid pictures over $\mathcal{P}$. Two monoid pictures $\mathbb{P}$ and $\mathbb{P}^{\prime}$ are equivalent relative to $\mathbf{Y}$ if there is a finite sequence of monoid pictures $\mathbb{P}=\mathbb{P}_{0}, \mathbb{P}_{1}, \ldots, \mathbb{P}_{m}=\mathbb{P}^{\prime}$ where, for $1 \leq i \leq m$, the monoid picture $\mathbb{P}_{i}$ is obtained from the picture $\mathbb{P}_{i-1}$ either by the insertion, deletion and replacement operations of the previous paragraph or else by inserting or deleting a subpicture of the form $W \cdot \mathbb{Y} \cdot V$ or of the form $W \cdot \mathbb{Y}^{-1} \cdot V$, where $W, V \in F(X)$ and $\mathbb{Y} \in \mathbf{Y}$. By definition, a set $\mathbf{Y}$ of spherical monoid pictures over $\mathcal{P}$ is a trivializer of $\mathcal{D}(\mathcal{P})$ if every spherical monoid picture is equivalent to an empty picture relative to $\mathbf{Y}$. By [7, Theorem 5.1], if $\mathbf{Y}$ is a trivializer for the Squier complex, then the elements of $\mathbf{Y}$ generate the first homology group of the Squier complex. The trivializer is also called a set of generating pictures. Some examples and more details of the trivializer can be found in [7-14].

For any monoid picture $\mathbb{P}$ over $\mathcal{P}$ and for any $R \in \mathbf{r}, \exp _{R}(\mathbb{P})$ denotes the exponent sum of $R$ in $\mathbb{P}$ which is the number of positive discs labeled by $R_{+}$, minus the number of negative discs labeled by $R_{-}$. For a non-negative integer $n, \mathcal{P}$ is said to be $n$-Cockcroft if $\exp _{R}(\mathbb{P}) \equiv 0$ $(\bmod n)$, (where congruence $(\bmod 0)$ is taken to be equality) for all $R \in \mathbf{r}$ and for all spherical pictures $\mathbb{P}$ over $\mathcal{P}$. Then a monoid $\mathcal{M}$ is said to be $n$-Cockcroft if it admits an $n$-Cockcroft presentation. In fact, to verify the $n$-Cockcroft property, it is enough to check for pictures $\mathbb{P} \in \mathbf{Y}$, where $\mathbf{Y}$ is a trivializer (see $[7,8]$ ). The 0 -Cockcroft property is usually just called Cockcroft. In general, we take $n$ to be equal to 0 or a prime $p$. Examples of monoid presentations with Cockcroft and $p$-Cockcroft properties can be found in [10].

Suppose that $\mathcal{P}=[X ; \mathbf{r}]$ is a finite presentation for a monoid $\mathcal{M}$. Then the Euler characteristic $\chi(\mathcal{P})$ is defined by $\chi(\mathcal{P})=1-|X|+|\mathbf{r}|$ and $\delta(\mathcal{M})$ is defined by $\delta(\mathcal{M})=1-$ $r k_{\mathbb{Z}}\left(H_{1}(\mathcal{M})\right)+d\left(H_{2}(\mathcal{M})\right)$. In unpublished work, Pride has shown that $\chi(\mathcal{P}) \geq \delta(\mathcal{M})$. With this background, we define the finite monoid presentation $\mathcal{P}$ to be efficient if $\chi(\mathcal{P})=\delta(\mathcal{M})$, and we define the monoid $\mathcal{M}$ to be efficient if it has an efficient presentation. Moreover, a presentation $\mathcal{P}_{0}$ for $\mathcal{M}$ is called minimal if $\chi\left(\mathcal{P}_{0}\right) \leq \chi(\mathcal{P})$ for all presentations $\mathcal{P}$ of $\mathcal{M}$. There is also interest in finding inefficient finitely presented monoids since if we can find 
a minimal presentation $\mathcal{P}_{0}$ for a monoid $\mathcal{M}$ such that $\mathcal{P}_{0}$ is not efficient, then we have $\chi\left(\mathcal{P}^{\prime}\right) \geq \chi\left(\mathcal{P}_{0}\right)>\delta(\mathcal{M})$ for all presentations $\mathcal{P}^{\prime}$ defining the same monoid $\mathcal{M}$. Thus, there is no efficient presentation for $\mathcal{M}$, that is, $\mathcal{M}$ is not an efficient monoid.

The following theorem was first given in [10]. (The group version of this result was proved by Epstein in [15].)

Theorem 1.1 Let $\mathcal{P}$ be a monoid presentation. Then $\mathcal{P}$ is efficient if and only if it is $p$ Cockcroft for some prime $p$.

Let $\mathcal{M}$ be a monoid with the presentation $\mathcal{P}=[X ; \mathbf{r}]$, and let

$$
P^{(l)}=\bigoplus_{S \in \mathbf{r}} \mathbb{Z} \mathcal{M} e_{S}
$$

be the free left $\mathbb{Z} \mathcal{M}$-module with basis $\left\{e_{S}: S \in \mathbf{r}\right\}$. For an atomic picture $\mathbb{A}=(U, S, \varepsilon, V)$ (where $U, V \in F(\mathbf{x}), S \in \mathbf{r}, \varepsilon= \pm 1$ ), we define eval ${ }^{(l)}(\mathbb{A})=\varepsilon \bar{U} e_{S} \in P^{(l)}$, where $\bar{U} \in \mathcal{M}(\mathcal{P})$. For any spherical monoid picture $\mathbb{P}$, we then define

$$
\operatorname{eval}^{(l)}(\mathbb{P})=\sum_{i=1}^{n} \operatorname{eval}^{(l)}\left(\mathbb{A}_{i}\right) \in P^{(l)}
$$

Let $\lambda_{\mathbb{P}, S}$ be the coefficient of $e_{S}$ in eval ${ }^{(l)}(\mathbb{P})$. So, we can write

$$
\operatorname{eval}^{(l)}(\mathbb{P})=\sum_{S \in \mathbf{r}} \lambda_{\mathbb{P}, S} e_{S} \in P^{(l)}
$$

Let $I_{2}^{(l)}(\mathcal{P})$ be a two-sided ideal of $\mathbb{Z} \mathcal{M}$ generated by the elements $\lambda_{\mathbb{P}, S}$, where $\mathbb{P}$ is a spherical monoid picture and $S \in \mathbf{r}$. Then this ideal is called the second Fox ideal of $\mathcal{P}$. More specifically, for a trivializer $\mathbf{Y}$ of $\mathcal{D}(\mathcal{P})$, the $\operatorname{set} I_{2}^{(l)}(\mathcal{P})$ is generated (as two-sided ideal) by the elements $\lambda_{\mathbb{P}, S}$, where $\mathbb{P} \in \mathbf{Y}$ and $S \in \mathbf{r}$. We note that all this above material given by the consideration 'left' can also be applied to 'right' for a monoid $\mathcal{M}$.

The definition and a standard presentation for the semi-direct product of two monoids can be found in $[10,11,14,16]$. Let $A$ and $K$ be arbitrary monoids with associated presentations $\mathcal{P}_{A}=[X ; \mathbf{r}]$ and $\mathcal{P}_{K}=[Y ; \mathbf{s}]$, respectively. Let $\mathcal{M}=K \rtimes_{\theta} A$ be the corresponding semi-direct product of these two monoids, where $\theta$ is a monoid homomorphism from $A$ to $\operatorname{End}(K)$. (We note that the reader can find some examples of monoid endomorphisms in [17].) The elements of $\mathcal{M}$ can be regarded as ordered pairs $(a, k)$ where $a \in A, k \in K$ with multiplication given by $(a, k)\left(a^{\prime}, k^{\prime}\right)=\left(a a^{\prime},\left(k \theta_{a^{\prime}}\right) k^{\prime}\right)$. The monoids $A$ and $K$ are identified with the submonoids of $\mathcal{M}$ having elements $(a, 1)$ and $(1, k)$, respectively. We want to define standard presentations for $\mathcal{M}$. For every $x \in X$ and $y \in Y$, choose a word, which we denote by $y \theta_{x}$, on $Y$ such that $\left[y \theta_{x}\right]=[y] \theta_{[x]}$ as an element of $K$. To establish notation, let us denote the relation $y x=x\left(y \theta_{x}\right)$ on $X \cup Y$ by $T_{y x}$ and write $\mathbf{t}$ for the set of relations $T_{y x}$. Then, for any choice of the words $y \theta_{x}$,

$$
\mathcal{P}_{\mathcal{M}}=[Y, X ; \mathbf{s}, \mathbf{r}, \mathbf{t}]
$$

is a standard monoid presentation for the semi-direct product $\mathcal{M}$. 
In [14], a finite trivializer set has been constructed for the standard presentation $\mathcal{P}_{\mathcal{M}}$, as given in (3), for the semi-direct product $\mathcal{M}$. We will essentially follow [10] in describing this trivializer set using spherical pictures and certain non-spherical subpictures of these.

If $W=y_{1} y_{2} \cdots y_{m}$ is a positive word on $Y$, then for any $x \in X$, we denote the word $\left(y_{1} \theta_{x}\right)\left(y_{2} \theta_{x}\right) \cdots\left(y_{m} \theta_{x}\right)$ by $W \theta_{x}$. If $U=x_{1} x_{2} \cdots x_{n}$ is a positive word on $X$, then for any $y \in Y$, we denote the word $\left(\cdots\left(\left(y \theta_{x_{1}}\right) \theta_{x_{2}}\right) \theta_{x_{3}} \cdots\right) \theta_{x_{n}}$ by $y \theta_{U}$, and this can be represented by a monoid picture, say $\mathbb{A}_{U, y}$, as in Figure 2(b). For $y \in Y$ and the relation $R_{+}=R_{-}$in the relation set $\mathbf{r}$, we have two important special cases, $\mathbb{A}_{R_{+}, y}$ and $\mathbb{A}_{R_{-}, y}$, of this consideration. We should note that these non-spherical pictures consist of only $T_{y x}$-discs $(x \in X)$. Let $S \in \mathbf{s}$ and $x \in X$. Since $\left[S_{+} \theta_{x}\right]_{\mathcal{P}_{K}}=\left[S_{-} \theta_{x}\right]_{\mathcal{P}_{K}}$, there is a non-spherical picture, say $\mathbb{B}_{S, x}$, over $\mathcal{P}_{K}$ with $\iota\left(\mathbb{B}_{S, x}\right)=S_{+} \theta_{x}$ and $\tau\left(\mathbb{B}_{S, x}\right)=S_{-} \theta_{x}$. Further, let $R_{+}=R_{-}$be a relation $R \in \mathbf{r}$ and $y \in Y$. Since $\theta$ is a homomorphism, by the definition on $y \theta_{U}$, we have that $y \theta_{R_{+}}$and $y \theta_{R_{-}}$ must represent the same element of the monoid $K$. That is, $\left[y \theta_{R_{+}}\right]_{\mathcal{P}_{K}}=\left[y \theta_{R_{-}}\right]_{\mathcal{P}_{K}}$. Hence, there is a non-spherical picture over $\mathcal{P}_{K}$ which we denote by $\mathbb{C}_{y, \theta_{R}}$ with $\iota\left(\mathbb{C}_{y, \theta_{R}}\right)=y \theta_{R_{+}}$and $\tau\left(\mathbb{C}_{y, \theta_{R}}\right)=y \theta_{R_{-}}$. In fact, there may be many different ways to construct the pictures $\mathbb{B}_{S, x}$ and $\mathbb{C}_{y, \theta_{R}}$. These pictures must exist, but they are not unique. On the other hand, the picture $\mathbb{A}_{U, y}$ will depend upon our choices for words $y \theta_{x}$, but this is unique once these choices are made.

After all, for $x \in X, y \in Y, R \in \mathbf{r}$ and $S \in \mathbf{s}$, one can construct spherical monoid pictures, say $\mathbb{P}_{S, x}$ and $\mathbb{P}_{R, y}$, by using the non-spherical pictures $\mathbb{B}_{S, x}, \mathbb{A}_{R_{+}, y}, \mathbb{A}_{R_{-}, y}$ and $\mathbb{C}_{y, \theta_{R}}$ (see Figures 2, 3 and 4 for the examples of these pictures). Let $\mathbf{X}_{\mathbf{A}}$ and $\mathbf{X}_{\mathbf{K}}$ be trivializer sets of $\mathcal{D}\left(\mathcal{P}_{A}\right)$ and $\mathcal{D}\left(\mathcal{P}_{K}\right)$, respectively. Also, let $\mathbf{C}_{\mathbf{1}}=\left\{\mathbb{P}_{S, x}: S \in \mathbf{s}, x \in X\right\}$ and $\mathbf{C}_{\mathbf{2}}=\left\{\mathbb{P}_{R, y}: R \in \mathbf{r}, y \in\right.$ $Y$ \}. Then, by $[10,14]$, it is known that for a presentation $\mathcal{P}_{\mathcal{M}}$, as in (3), a trivializer set of $\mathcal{D}\left(\mathcal{P}_{\mathcal{M}}\right)$ is $\mathbf{X}_{\mathcal{M}}=\mathbf{X}_{\mathbf{A}} \cup \mathbf{X}_{\mathbf{K}} \cup \mathbf{C}_{\mathbf{1}} \cup \mathbf{C}_{\mathbf{2}}$.

\section{Generators over the semi-direct product of finite cyclic monoids}

In fact, this is the main section of the paper and it will be given as two subsections under the names of Part I and Part II. Since we will define generating functions by considering the exponent sums of the generating pictures over the presentation of this semi-direct product, the first subsection is aimed to define these generating pictures and the related results about them.

\subsection{Part I: generating pictures}

In this subsection, we will mainly present the efficiency (equivalently, $p$-Cockcroft property for a prime $p$ by Theorem 1.1) for the semi-direct products of finite cyclic monoids.

Let $A$ and $K$ be two finite cyclic monoids with presentations

$$
\mathcal{P}_{A}=\left[x ; x^{\mu}=x^{\lambda}\right] \text { and } \mathcal{P}_{K}=\left[y ; y^{k}=y^{l}\right]
$$

respectively, where $l, k, \lambda, \mu \in \mathbb{Z}^{+}$such that $l<k$ and $\lambda<\mu$, or equivalently,

$$
\mu=\lambda+r \quad(1 \leq r \leq \mu-1) \quad \text { and } \quad k=l+\omega \quad(1 \leq \omega \leq k-1) .
$$

Due to [10], a trivializer set $\mathbf{X}_{\mathbf{K}}$ (and similarly $\mathbf{X}_{\mathbf{A}}$ ) of the Squier complex $\mathcal{D}\left(\mathcal{P}_{K}\right.$ ) (and similarly $\left.\mathcal{D}\left(\mathcal{P}_{A}\right)\right)$ is given by the pictures $\mathbb{P}_{k, l}^{m}(1 \leq m \leq k-1)$, as in Figure 1 .

Let $\psi_{i}(0 \leq i \leq k-1)$ be an endomorphism of $K$. Then we have a mapping $x \rightarrow \operatorname{End}(K)$, $x \longmapsto \psi_{i}$. In fact this induces a homomorphism $\theta: A \longrightarrow \operatorname{End}(K), x \mapsto \psi_{i}$ if and only if 
$\psi_{i}^{\mu}=\psi_{i}^{\lambda}$. Since $\psi_{i}^{\mu}$ and $\psi_{i}^{\lambda}$ are equal if and only if they agree on the generator $y$ of $K$, we must have

$$
\left[y^{i^{\mu}}\right]=\left[y^{i^{\lambda}}\right]
$$

We then have the semi-direct product $M=K \rtimes_{\theta} A$ and, by [10], a standard presentation

$$
\mathcal{P}_{M}=\left[y, x ; S, R, T_{y x}\right]
$$

as in (3), for the monoid $M$ where

$$
S: y^{k}=y^{l}, \quad R: x^{\mu}=x^{\lambda} \quad \text { and } \quad T_{y x}: y x=x y^{i} .
$$

In the rest of the paper, we will assume that the equality in Equation (6) holds when we talk about the semi-direct product $M$ of $K$ by $A$.

The subpicture $\mathbb{B}_{S, x}$ can be drawn as in Figure 2(a), and in fact, by considering this subpicture, we clearly have

$$
\exp _{S}\left(\mathbb{B}_{S, x}\right)=i
$$

As it is seen in Figure 2(b), we also have the subpicture $\mathbb{A}_{R_{+}, y}$ (and similarly $\mathbb{A}_{R_{-}, y}$ ) with

$$
\exp _{T_{y x}}\left(\mathbb{A}_{R_{+}, y}\right)=1+i+i^{2}+\cdots+i^{\mu-1}=\frac{i^{\mu}-1}{i-1}
$$

and

$$
\exp _{T_{y x}}\left(\mathbb{A}_{R_{-}, y}\right)=1+i+i^{2}+\cdots+i^{\lambda-1}=\frac{i^{\lambda}-1}{i-1} .
$$

By equality (6), we must have $\left[y^{i^{\mu}}\right]=\left[y^{i^{\lambda}}\right]$. Hence, by [10], the subpicture $\mathbb{C}_{y, \theta_{R}}$ with

$$
\iota\left(\mathbb{C}_{y, \theta_{R}}\right)=y^{i^{\mu}}, \quad \tau\left(\mathbb{C}_{y, \theta_{R}}\right)=y^{i^{\lambda}} \quad \text { and } \quad \exp _{S}\left(\mathbb{C}_{y, \theta_{R}}\right)=\frac{i^{\mu}-i^{\lambda}}{k-l}
$$

can be depicted as in Figure 3.

After all, the whole generating pictures $\mathbb{P}_{S, x}$ and $\mathbb{P}_{R, y}$ can be drawn as in Figure 4.

The following result states necessary and sufficient conditions for the presentation of the split extension of two finite monogenic monoids to be efficient.

Proposition 2.1 ([18]) Let $p$ be a prime. Suppose that $K \rtimes_{\theta} A$ is a monoid with the associated monoid presentation $\mathcal{P}_{M}$, as in (7). Then $\mathcal{P}_{M}$ is p-Cockcroft (equivalently efficient) if and only if

$$
p|k-l, \quad p| i-1, \quad p\left|\frac{i^{\mu}-i^{\lambda}}{k-l}, \quad p\right| \frac{i^{\mu}-i^{\lambda}}{i-1} .
$$

Remark 2.2 To be an example of Proposition 2.1, one can take

- $k=10, l=6, \mu=4, \lambda=2$ or $k=6, l=2, \mu=5, \lambda=3$ while $p=2$ and $i=3$ to get

2 -Cockcroft property for the presentation $\mathcal{P}_{M}$ in (7), or more generally 
- for any prime $p, k=(p+1)\left[\frac{(p+1)^{p}-1}{p}\right]+1, l=1, \mu=p+1=i$ and $\lambda=1$ to get $p$-Cockcroft property for the presentation $\mathcal{P}_{M}$ in (7).

Considering Theorem 1.1, one can say that the monoid presentation $\mathcal{P}_{M}$, as in (7), is efficient if and only if there is a prime $p$ such that

$$
\begin{aligned}
& \exp _{y}(S) \equiv 0(\bmod p), \quad \exp _{S}\left(\mathbb{B}_{S, x}\right) \equiv 1(\bmod p), \\
& \exp _{T_{y x}}\left(\mathbb{C}_{y, \theta_{R}}\right) \equiv 0(\bmod p), \quad \exp _{T_{y x}}\left(\mathbb{A}_{R, y}\right) \equiv 0(\bmod p) .
\end{aligned}
$$

In particular, if we choose $\exp _{S}\left(\mathbb{B}_{S, x}\right)=i=0$ or 2 , then $\mathcal{P}_{M}$ will be inefficient.

Recall that, by the meaning of finite cyclic monoids, $\exp _{y}(S)=k-l$ cannot be equal to 0 . We also note that a similar proof for the following result about minimal but inefficiency of $\mathcal{P}_{M}$ can be found in [18].

Proposition 2.3 Let $M$ be the semi-direct product of $K$ by $A$, and let $\mathcal{P}_{M}$, as in (7), be the presentation for $M$ where $l, k, \lambda, \mu, i \in \mathbb{Z}^{+}$and $l<k, \lambda<\mu$. If $i=2$ and the subtraction $k-l$ is not even and not equal to 1 , then $\mathcal{P}_{M}$ is minimal but inefficient.

Remark 2.4 To be an example of Proposition 2.3, we can consider the following:

- For an odd positive integer $t$, one can take $k=4 t, l=t, \mu=3 t, \lambda=t$ and $i=2$ in the presentation given in (7). Since $k-l=3 t \neq 2 n\left(n \in \mathbb{Z}^{+}\right)$, the presentation is minimal but inefficient.

- For all $s, t \in \mathbb{Z}^{+}$such that $s<t$, one can take $k=2 t+1, l=2 s, \mu=k-l, \lambda=1$ and $i=2$ in the presentation given in (7). Since $k-l=2(t-s)+1 \neq 2 n\left(n \in \mathbb{Z}^{+}\right)$, the presentation is minimal while it is inefficient.

\subsection{Part Il: generating functions}

By considering the pictures defined in the previous section and also the evaluations obtained from them, we will define the related generating functions. In another words, by taking into account Propositions 2.1 and 2.3, we will reach our main aim over monoids of this paper.

We firstly recall that, as noted in [4, Remark 1.1], if a monoid presentation satisfies efficiency or inefficiency (while it is minimal), then it always has a minimal number of generators. Working with the minimal number of elements gives a great opportunity to define related generating functions over this presentation. This will be one of the key points in our results.

Our first result of this section is related to the connection of the monoid presentation in (7) with array polynomials. In fact array polynomials $S_{a}^{n}(x)$ are defined by means of the generating function

$$
\frac{\left(e^{t}-1\right)^{a} e^{t x}}{x !}=\sum_{n=0}^{\infty} S_{a}^{n}(x) \frac{t^{n}}{n !}
$$

(cf. [19-21]). According to the same references, array polynomials can also be defined as the form

$$
S_{a}^{n}(x)=\frac{1}{a !} \sum_{j=0}^{a}(-1)^{a-j}\left(\begin{array}{l}
a \\
j
\end{array}\right)(x+j)^{n} .
$$


Since the coefficients of array polynomials are integers, they find very large application area, especially in system control ( $c f$. [22]). In fact, these integer coefficients give us the opportunity to use these polynomials in our case. We should note that there also exist some other polynomials, namely Dickson, Bell, Abel, Mittag-Leffler etc., which have integer coefficients which will not be handled in this paper.

From (5), we know that $\mu=\lambda+r$, where $1 \leq r \leq \mu-1$. Hence, by considering the meaning and conditions of Proposition 2.1, we obtain the following theorem as one of the main results of this paper.

Theorem 2.5 The efficient presentation $\mathcal{P}_{M}$ defined in (7) has a set of generating functions

$$
\left.\begin{array}{l}
p_{1}(x)=S_{n}^{n}(x)-i S_{0}^{1}(x), \quad p_{2}(y)=(k-l) S_{n}^{n}(y), \\
p_{3}(x)=\frac{i^{\lambda}\left(i^{r}-1\right)}{i-1} S_{n}^{n}(x), \quad p_{4}(y)=\frac{i^{\lambda}\left(i^{r}-1\right)}{k-l} S_{n}^{n}(y),
\end{array}\right\}
$$

where $S_{a}^{n}(x)$ and $S_{a}^{n}(y)$ are defined as in (8).

Proof Let us consider the generating pictures $\mathbb{P}_{S, x}, \mathbb{P}_{R, y}$ (in Figure 4) with their nonspherical subpictures defined in Figures 2 and 3, and the generating pictures of finite monogenic monoids defined in Figure 1. Recall that by counting the exponent sums of the discs $R, S$ and $T_{y x}$ in the related pictures, the conditions of Proposition 2.1 have been obtained [18]. (For more similar results and applications, one can see the papers [10,11].)

To reach our aim in the proof, we first need to calculate eval ${ }^{(l)}\left(\mathbb{P}_{S, x}\right)$, eval ${ }^{(l)}\left(\mathbb{P}_{R, y}\right)$, $\operatorname{eval}^{(l)}\left(\mathbb{P}_{k, l}^{m}\right)(1 \leq m \leq k-1)$ and eval ${ }^{(l)}\left(\mathbb{P}_{\lambda+r, \lambda}^{n}\right)(1 \leq n \leq(\lambda+r)-1)$. By Equations (1) and (2), we have

$$
\begin{aligned}
& \operatorname{eval}^{(l)}\left(\mathbb{P}_{S, x}\right)=\left(1-x\left(\operatorname{eval}^{(l)}\left(\mathbb{B}_{S, x}\right)\right)\right) e_{S}+\left(\frac{\partial^{M} S}{\partial y}\right) e_{T_{y x}}, \\
& \operatorname{eval}^{(l)}\left(\mathbb{P}_{R, y}\right)=\left(\operatorname{eval}^{(l)}\left(\mathbb{A}_{R_{+}, y}\right)-\operatorname{eval}^{(l)}\left(\mathbb{A}_{R_{-}, y}\right)\right) e_{T_{y x}}+(1-y) e_{R}+\left(\operatorname{eval}^{(l)}\left(\mathbb{C}_{y, \theta_{R}}\right)\right) e_{S},
\end{aligned}
$$

where $\frac{\partial}{\partial y}$ denotes the Fox derivation [23]. Also, for each $1 \leq m \leq k-1$ and $1 \leq n \leq(\lambda+$ $r)-1$,

$$
\operatorname{eval}^{(l)}\left(\mathbb{P}_{k, l}^{m}\right)=\left(1-y^{k-m}\right) e_{S} \quad \text { and } \quad \operatorname{eval}^{(l)}\left(\mathbb{P}_{\lambda+r, \lambda}^{n}\right)=\left(1-x^{(\lambda+r)-n}\right) e_{R}
$$

Therefore, by the definition, the second Fox ideal $I_{2}^{(l)}\left(\mathcal{P}_{M}\right)$ of the presentation $\mathcal{P}_{M}$ in (7) is generated by the polynomial elements

$$
\left.\begin{array}{l}
1-x\left(\operatorname{eval}^{(l)}\left(\mathbb{B}_{S, x}\right)\right), \quad \frac{\partial^{M} S}{\partial y}, \\
\operatorname{eval}^{(l)}\left(\mathbb{A}_{R_{+}, x}\right)-\operatorname{eval}^{(l)}\left(\mathbb{A}_{R_{-}, x}\right), \\
1-y^{k-1}, 1-y^{k-2}, \ldots, 1-y, \quad \operatorname{eval}^{(l)}\left(\mathbb{C}_{y, \theta_{R}}\right), \\
1-x^{(\lambda+r)-1}, 1-x^{(\lambda+r)-2}, \ldots, 1-x .
\end{array}\right\}
$$

We need to keep our calculations going to other evaluations in the above polynomial elements. To do that, one can consider the augmentation map aug : $\mathbb{Z} M \longrightarrow \mathbb{Z}, b \longmapsto 1$. 
Under this map, it is easy to see that

$$
\left.\begin{array}{l}
\operatorname{aug}\left(\operatorname{eval}^{(l)}\left(\mathbb{B}_{S, x}\right)\right)=\exp _{S}\left(\mathbb{B}_{S, x}\right)=i, \\
\operatorname{aug}\left(\frac{\partial^{M} S}{\partial y}\right)=\exp _{y}(S)=k-l \\
\operatorname{aug}\left(\operatorname{eval}^{(l)}\left(\mathbb{A}_{R_{+}, y}\right)-\operatorname{eval}^{(l)}\left(\mathbb{A}_{R_{-}, y}\right)\right)=\exp _{T_{y x}}\left(\mathbb{P}_{R, y}\right)=\frac{i^{\lambda+r}-i^{\lambda}}{i-1}, \\
\operatorname{aug}\left(\operatorname{eval}^{(l)}\left(\mathbb{C}_{y, \theta_{R}}\right)\right)=\exp _{S}\left(\mathbb{P}_{R, y}\right)=\frac{i^{\lambda+r}-i^{\lambda}}{k-l}
\end{array}\right\}
$$

and for each $1 \leq m \leq k-1$ and $1 \leq n \leq(\lambda+r)-1$,

$$
\operatorname{aug}\left(\operatorname{eval}^{(l)}\left(\mathbb{P}_{k, l}^{m}\right)\right)=0 \quad \text { and } \quad \operatorname{aug}\left(\operatorname{eval}^{(l)}\left(\mathbb{P}_{\lambda+r, \lambda}^{n}\right)\right)=0
$$

Now, by using (8) and keeping in our mind the coefficients of array polynomials are integer, we clearly have

$$
S_{a}^{n}(b)= \begin{cases}b^{n} ; & a=0, \\ b ; & a=0 \text { and } n=1, \\ 1 ; & k=n \text { or } n=a=0 .\end{cases}
$$

Then, by reformulating the elements in (10) and (11) of the second Fox ideal $I_{2}^{(l)}\left(\mathcal{P}_{M}\right)$, we arrive at the functions in (9) as desired.

Considering Remark 2.2, we obtain the following corollary as a consequence of Theorem 2.5 .

Corollary 2.6 For any prime p, the presentation

$$
\mathcal{P}_{M}=\left[y, x ; y^{(p+1)\left[\frac{(p+1)^{p}-1}{p}\right]+1}=y, x^{p+1}=x, y x=x y^{p+1}\right]
$$

has a set of generating functions

$$
\begin{aligned}
& p_{1}(x)=S_{n}^{n}(x)-(p+1) S_{0}^{1}(x), \quad p_{2}(y)=(p+1)\left[\frac{(p+1)^{p}-1}{p}\right] S_{n}^{n}(y), \\
& p_{3}(x)=\frac{(p+1)\left[(p+1)^{p}-1\right]}{p} S_{n}^{n}(x), \quad p_{4}(y)=p S_{n}^{n}(y) .
\end{aligned}
$$

In Proposition 2.3, the minimality (while satisfying inefficiency) of the presentation $\mathcal{P}_{M}$ was expressed in (7). Thus, by considering the meaning and conditions of Proposition 2.3, we obtain the following theorem as another main result of this paper. Since the proof is quite similar to the proof of Theorem 2.5, we omit it.

Theorem 2.7 The inefficient but minimal presentation $\mathcal{P}_{M}$ defined in (7) has a set of generating functions

$$
\begin{array}{ll}
p_{1}(x)=S_{n}^{n}(x)-2 S_{0}^{1}(x), & p_{2}(y)=(k-l) S_{n}^{n}(y), \\
p_{3}(x)=2^{\lambda}\left(2^{r}-1\right) S_{n}^{n}(x), & p_{4}(y)=\frac{2^{\lambda}\left(2^{r}-1\right)}{k-l} S_{n}^{n}(y),
\end{array}
$$

where $k-l$ is an odd integer and $S_{a}^{n}(x)$ and $S_{a}^{n}(y)$ are defined as in (8). 
By considering Remark 2.4, we can have the following consequences of Theorem 2.7.

Corollary 2.8 For an odd positive integer $t$, the presentation

$$
\mathcal{P}_{M}=\left[y, x ; y^{4 t}=y^{t}, x^{3 t}=x^{t}, y x=x y^{2}\right]
$$

has a set of generating functions

$$
\begin{array}{ll}
p_{1}(x)=S_{n}^{n}(x)-2 S_{0}^{1}(x), & p_{2}(y)=3 t S_{n}^{n}(y), \\
p_{3}(x)=2^{t}\left(2^{2 t}-1\right) S_{n}^{n}(x), & p_{4}(y)=\frac{2^{t}\left(2^{2 t}-1\right)}{3 t} S_{n}^{n}(y) .
\end{array}
$$

Corollary 2.9 For any positive integers $s$ and $t$ with the condition $s<t$, the presentation

$$
\mathcal{P}_{M}=\left[y, x ; y^{2 t+1}=y^{2 s}, x^{2(t-s)+1}=x, y x=x y^{2}\right]
$$

has a set of generating functions

$$
\begin{aligned}
& p_{1}(x)=S_{n}^{n}(x)-2 S_{0}^{1}(x), \quad p_{2}(y)=[2(t-s)+1] S_{n}^{n}(y) \\
& p_{3}(x)=2\left[2^{2(t-s)}-1\right] S_{n}^{n}(x), \quad p_{4}(y)=\frac{2\left[2^{2(t-s)}-1\right]}{2(t-s)+1} S_{n}^{n}(y) .
\end{aligned}
$$

Remark 2.10 Since both presentations in Propositions 2.1 and 2.3 have the minimal number of generators because of their efficiency or inefficiency (but minimal) status, this situation affected very positively the number and type of generating functions defined on them.

At this point, we should note that for $t_{1} \neq t_{2} \in \mathbb{R}^{+}, \gamma \in \mathbb{C}, a \in \mathbb{N}_{0}$, generalized array type polynomials $\mathcal{S}_{a}^{n}\left(x ; t_{1}, t_{2} ; \gamma\right)$ related to the non-negative real parameters have been recently developed (in [20]) and some elementary properties including recurrence relations of these polynomials have been derived. In fact, by setting $t_{1}=\gamma=1$ and $t_{2}=e$, Equation (8) is obtained.

Remark 2.11 For a future project, one can study the generalization of Theorems 2.5 and 2.7 by using $\mathcal{S}_{a}^{n}\left(x ; t_{1}, t_{2} ; \gamma\right)$.

The remaining goal of this section is to make a connection between the presentation $\mathcal{P}_{M}$ in (7) and Stirling numbers of the second kind (cf. [20, 24-28] and the references of these papers). In fact, Stirling numbers of the second kind $S(n, a)$ are defined by means of the generating function

$$
\frac{\left(e^{t}-1\right)^{a}}{a !}=\sum_{n=0}^{\infty} S(n, a) \frac{t^{n}}{n !}
$$

(see $[27,28])$. According to [20, Theorem 1, Remark 2], Stirling numbers can also be defined as the form

$$
S(n, a)=\frac{1}{a !} \sum_{j=0}^{a}(-1)^{j}\left(\begin{array}{l}
a \\
j
\end{array}\right)(k-j)^{n} .
$$


We remind that these numbers satisfy the well-known properties

$$
S(n, a)= \begin{cases}1 ; & a=1 \text { or } a=n \\
\left(\begin{array}{l}
n \\
2
\end{array}\right) ; & a=n-1, \\
\delta_{n, 0} ; & a=0,\end{cases}
$$

where $\delta_{n, 0}$ denotes the Kronecker symbol (see [27, 28]). It is known that Stirling numbers are used in combinatorics, in number theory, in discrete probability distributions for finding higher-order moments, etc. We finally note that since $S(n, a)$ is the number of ways to partition a set of $n$ objects into $k$ groups, these numbers find an application area in combinatorics and in theory of partitions.

In addition to the above formulas, for $S(n, a)$, by [20,26, 27], we also have

$$
x^{n}=\sum_{a=0}^{n}\left(\begin{array}{l}
x \\
a
\end{array}\right) a ! S(n, a)
$$

as a formula for Stirling numbers. Therefore, by taking $n=1$ and $n=0$ in Equation (12), the polynomial elements of the second Fox ideal $I_{2}^{(l)}\left(\mathcal{P}_{M}\right)$ of the presentation $\mathcal{P}_{M}$ in (7) can be restated as follows:

$$
\left.\begin{array}{l}
x^{0}-i x^{1}=\sum_{a=0}^{0}\left(\begin{array}{c}
x \\
a
\end{array}\right) a ! S(0, a)-i \sum_{a=0}^{1}\left(\begin{array}{c}
x \\
a
\end{array}\right) a ! S(1, a), \\
(k-l) y^{0}=(k-l) \sum_{a=0}^{0}\left(\begin{array}{c}
y \\
a
\end{array}\right) a ! S(0, a), \\
\frac{i^{\lambda}\left(i^{r}-1\right)}{i-1} x^{0}=\frac{i^{\lambda}\left(i^{r}-1\right)}{i-1} \sum_{a=0}^{0}\left(\begin{array}{c}
x \\
a
\end{array}\right) a ! S(0, a), \\
\frac{i^{\lambda}\left(i^{r}-1\right)}{k-l} y^{0}=\frac{i^{\lambda}\left(i^{r}-1\right)}{k-l} \sum_{a=0}^{0}\left(\begin{array}{c}
y \\
a
\end{array}\right) a ! S(0, a) .
\end{array}\right\}
$$

After that, as a different version of Theorem 2.5 (and so Theorem 2.7), we present the following result.

Theorem 2.12 The efficient presentation $\mathcal{P}_{M}$ in (7) has a set of generating functions in terms of Stirling numbers as given in (13). By taking $i=2$ and $k-l$ is an odd positive integer, we get a set of generating functions in terms of Stirling numbers for the inefficient but minimal presentation of the form as defined in (7).

Furthermore, in a recent work, Simsek [20] has constructed the generalized $\gamma$-Stirling numbers of the second kind $\mathcal{S}(n, v ; a, b ; \gamma)$ related to non-negative real parameters $(a, b \in$ $\mathbb{R}^{+}, a \neq b$, a complex number $\gamma$ and $v \in \mathbb{N}_{0}$ ). In fact, this new generalization is defined by the generating function as the equality

$$
f_{S, v}(t ; a, b ; \gamma)=\frac{\left(\gamma b^{t}-a^{t}\right)^{v}}{v !}=\sum_{n=0}^{\infty} \mathcal{S}(n, v ; a, b ; \gamma) \frac{t^{n}}{n !}
$$

By setting $a=1$ and $b=e$ in (14), one can obtain the $\gamma$-Stirling numbers of the second kind $S(n, v ; \gamma)$ which are defined by the generating function

$$
\frac{\left(\gamma e^{t}-1\right)^{v}}{\nu !}=\sum_{n=0}^{\infty} S(n, v ; \gamma) \frac{t^{n}}{n !}
$$


(see $[27,28])$. According to the same references, by substituting $\gamma=1$ into the above equation, the Stirling numbers of the second kind $S(n, v)$ are obtained.

By considering this new generalization $\mathcal{S}(n, v ; a, b ; \gamma)$, in [20, Theorem 1$]$, the equality

$$
\mathcal{S}(n, v ; a, b ; \gamma)=\frac{1}{v !} \sum_{j=0}^{n}(-1)^{j}\left(\begin{array}{l}
v \\
j
\end{array}\right) \gamma^{\nu-j}(j \ln a+(v-j) \ln b)^{n}
$$

has also been obtained for $\gamma$-Stirling numbers of the second kind. In fact, by setting $a=1$ and $b=e$ in (15), one can get the following equality on $\gamma$-Stirling numbers:

$$
S(n, v ; \gamma)=\frac{1}{v !} \sum_{j=0}^{v}\left(\begin{array}{l}
v \\
j
\end{array}\right) \lambda^{(v-j)}(-1)^{j}(v-j)^{n}
$$

(see $[27,28])$.

Hence, we can present the following note.

Remark 2.13 It is clearly seen that Stirling numbers have been only considered in Theorems 2.5 and 2.7 (and the corollaries about them). However, one can also study the $\gamma$-Stirling numbers $S(n, v ; \gamma)$ defined in (16) and generalized $\gamma$-Stirling numbers $\mathcal{S}(n, v ; a, b ; \gamma)$ defined in (15) to obtain different types of generating functions.

\section{The constant function related to main results}

In Theorems 2.5, 2.7 and 2.12, we have actually used

$$
\frac{i^{\lambda}\left(i^{r}-1\right)}{i-1} \text { and } \frac{i^{\lambda}\left(i^{r}-1\right)}{k-l}
$$

as the constants of defined generating functions. In this section, by representing these constants as a single function (see Equation (17) below), we investigate some new properties over it.

Hence, let us consider the analytic function

$$
f(z, r, \lambda, k, l)=\frac{z^{\lambda+r}-z^{\lambda}}{k-l}
$$

where $z \in \mathbb{C}$ and $r, \lambda, k, l \in \mathbb{Z}^{+}$. To reach our aim, let us first replace the complex element $z$ by a positive integer $i$ in (17), and then apply some fundamental algebraic progress to it. Therefore,

$$
\begin{aligned}
f(i, r, \lambda, k, l) & =\frac{i^{\lambda+r}-i^{\lambda}}{k-l}=\frac{i^{\lambda}}{k-l}\left(i^{r}-1\right) \\
& =\frac{i^{\lambda}}{k-l}(i-1)\left(i^{r-1}+\cdots+1\right)=\frac{i^{\lambda}}{k-l}(i-1) \phi_{r-1}(i),
\end{aligned}
$$

where $\phi_{r-1}(i)$ is a cyclotomic polynomial having degree $r-1$. By considering finite powers of the function $f(z, r, \lambda, k, l)$ given in (17), we can get

$$
Y(z)=[f(z, r, \lambda, k, l)]^{m}=\frac{m !}{m !}\left(\frac{z^{\lambda+r}-z^{\lambda}}{k-l}\right)^{m},
$$

which is actually $m$-times algebraic multiplication of the function $f$. 
Now, if we replace $z$ by $e^{t}$, then we get

$$
\begin{aligned}
Y\left(e^{t}\right) & =\frac{m !}{m !}\left(\frac{e^{t(\lambda+r)}-e^{t \lambda}}{k-l}\right)^{m} \\
& =\frac{m !}{k-l} e^{m t \lambda} \frac{\left(e^{t r}-1\right)^{m}}{m !} \\
& =\frac{m !}{k-l} e^{m t \lambda} \sum_{n=0}^{\infty} S(n, m) r^{n} \frac{t^{n}}{n !},
\end{aligned}
$$

where $S(n, m)$ defines the Stirling numbers of the second-kind

$$
=\frac{m !}{k-l} \sum_{n=0}^{\infty} m^{n} \lambda^{n} \frac{t^{n}}{n !} \sum_{n=0}^{\infty} S(n, m) r^{n} \frac{t^{n}}{n !} \text {. }
$$

Further, by applying the Cauchy multiplication in (21), we finally obtain

$$
Y\left(e^{t}\right)=\frac{m !}{k-l} \sum_{n=0}^{\infty}\left[\sum_{a=0}^{n}\left(\begin{array}{l}
n \\
a
\end{array}\right) S(a, m) r^{a} m^{n-a} \lambda^{n-a}\right] \frac{t^{n}}{n !} .
$$

All these above processes imply the following result.

\section{Theorem 3.1}

$$
\left(f\left(e^{t}, r, \lambda, k, l\right)\right)^{m}=\sum_{n=0}^{\infty} \frac{a_{n}}{k-l} \frac{t^{n}}{n !}
$$

where

$$
a_{n}=m ! \sum_{a=0}^{n}\left(\begin{array}{l}
n \\
a
\end{array}\right) S(a, m) r^{a} m^{n-a} \lambda^{n-a}
$$

and $S(a, m)$ denotes the Stirling numbers of second kind.

Some properties of the function $Y(z)=[f(z, r, \lambda, k, l)]^{m}$ in (19) can be expressed as follows:

- If $z \in \mathbb{C}$, then $Y(z)$ is an analytic function, and then it has a power series as defined in the above theorem with Equation (22).

- If $z \in \mathbb{R}$, then $Y(z)$ is a continuous function which is actually a polynomial function having degree $\lambda+r$.

- If we replace $z$ by $e^{t}$ in (19), we obtain the second kind Stirling numbers.

Remark 3.2 Setting $m=1$ in (22), one can easily see that

$$
f\left(e^{t}, r, \lambda, k, l\right)=\sum_{n=0}^{\infty}\left[\sum_{a=0}^{n} \frac{\left(\begin{array}{l}
n \\
a
\end{array}\right) r^{a} \lambda^{n-a}}{k-l}\right] \frac{t^{n}}{n !},
$$

since $S(n, 1)=1$. 
By considering [20, Eq. (3.2)] and Equation (20), we can extend Remark 3.2 to a general natural number $m>1$ as in the following theorem.

\section{Theorem 3.3}

$$
\left(\frac{e^{t(\lambda+r)}-e^{t \lambda}}{k-l}\right)^{m}=\frac{m !}{k-l} \sum_{n=0}^{\infty} \mathcal{S}_{m}^{n}(m \lambda) r^{n} \frac{t^{n}}{n !}
$$

where $\mathcal{S}_{m}^{n}(m \lambda)$ denotes the array polynomials.

As it was seen, only the function defined in (18) itself is enough to represent almost all the conditions in Propositions 2.1 and 2.3. Thus, we can express the following remark which depicts some new studying areas for a future project.

\section{Remark 3.4}

- If we replace $z$ by $i$, then we can study the changes on the generating pictures defined in Figures 1, 2, 3 and 4. By playing on this function, one can hope to apply some operations (as defined in $[7,8]$ ) on the pictures, and so it could happen to represent

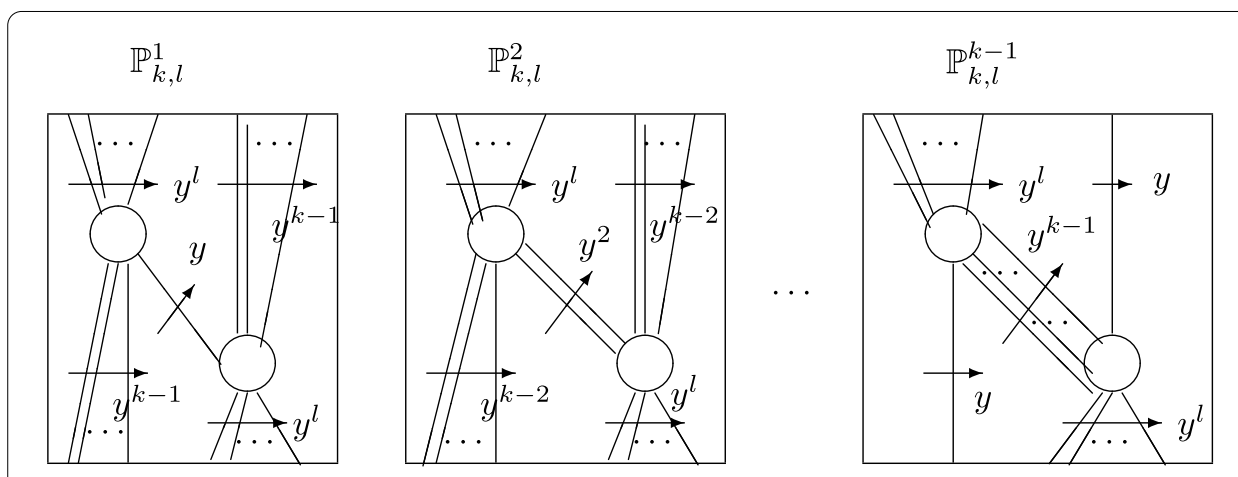

Figure 1 Generating pictures of finite monogenic monoids.

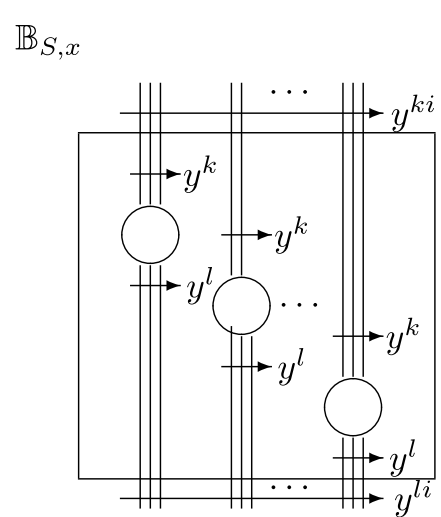

(a)
$\mathbb{A}_{R_{+}, y}$

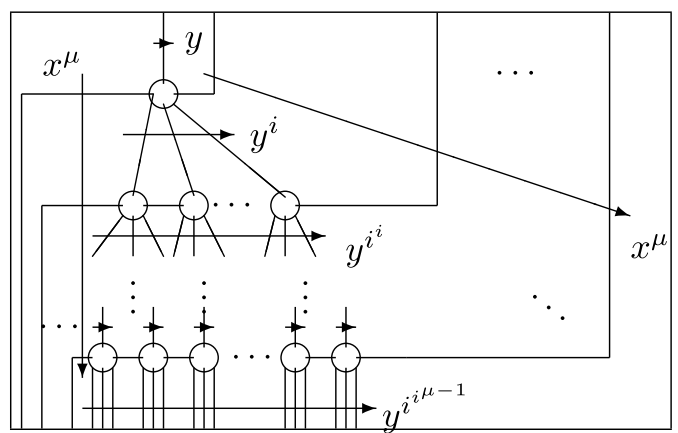

(b)

Figure 2 Two subpictures of the generating pictures. 


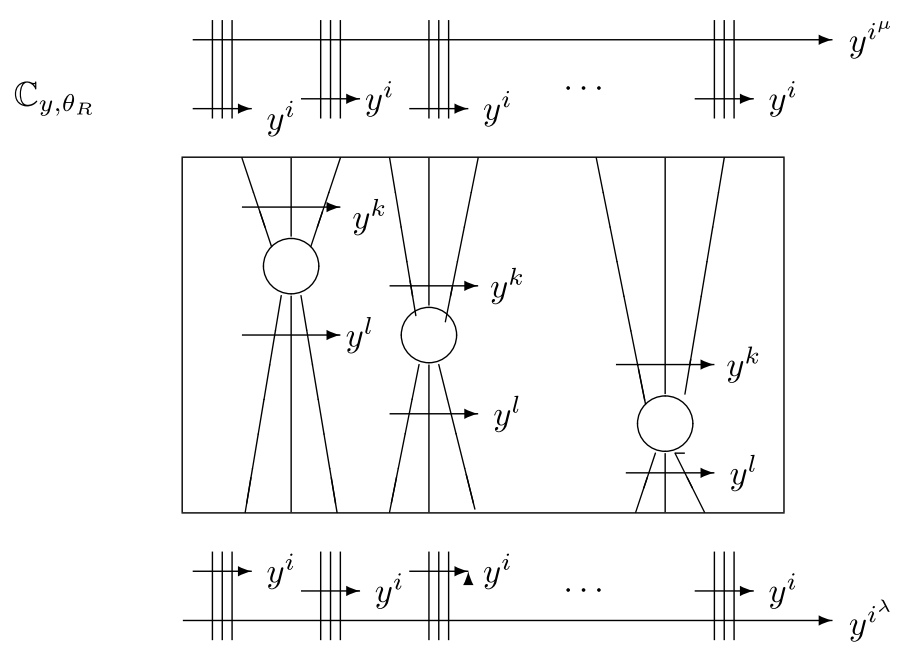

Figure 3 Subpicture $\mathbb{C}_{y, \theta_{R}}$ of the generating picture.

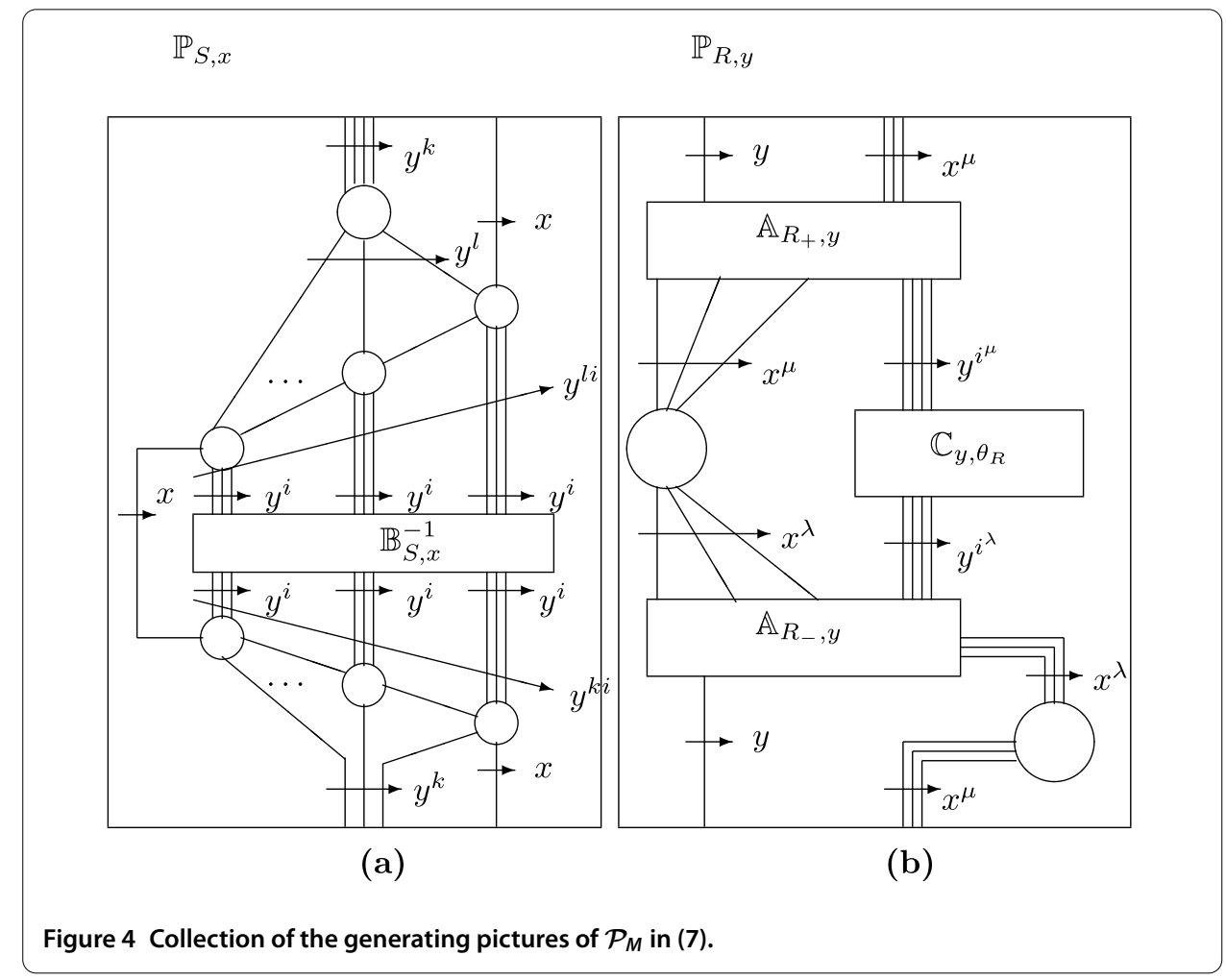

these algebraic operations by generating functions to obtain efficiency or inefficiency (while minimality holds).

- While $z \in \mathbb{C}$ and $z \in \mathbb{R}$, analytic and functional equations can be studied.

- As we have partially done in the above, replacing $z$ by $e^{t}$, one can study the generating functions of array polynomials and Stirling numbers. 


\subsection{Some other properties over this constant}

Let us consider the first derivation of the function in (17). We then have

$$
\begin{aligned}
f^{\prime}(z, r, \lambda, k, l) & =\frac{(\lambda+r) z^{\lambda+r-1}-\lambda z^{\lambda-1}}{k-l}=\frac{\lambda z^{\lambda+r-1}-\lambda z^{\lambda-1}}{k-l}+\frac{r z^{\lambda+r-1}}{k-l} \\
& =\frac{\lambda z^{\lambda-1}}{k-l}\left(z^{r}-1\right)+\frac{r z^{\lambda+r-1}}{k-l}
\end{aligned}
$$

or equivalently,

$$
f^{\prime}(z, r, \lambda, k, l)=\frac{\lambda z^{\lambda-1}}{k-l}(z-1) \phi_{r-1}(z)+\frac{r z^{\lambda+r-1}}{k-l} .
$$

In (23), replacing $z$ by $i$, we get

$$
f^{\prime}(i, r, \lambda, k, l)=\frac{\lambda i^{\lambda-1}}{k-l}(i-1) \phi_{r-1}(i)+\frac{r i^{\lambda+r-1}}{k-l}=\lambda\left[\frac{i^{\lambda-1}}{k-l}(i-1) \phi_{r-1}(i)\right]+\frac{r i^{\lambda+r-1}}{k-l},
$$

and then by using (18), we have

$$
f^{\prime}(i, r, \lambda, k, l)=\frac{\lambda}{i} f(i, r, \lambda, k, l)+\frac{r i^{\lambda+r-1}}{k-l} .
$$

As the next step, let us calculate the second derivative of $f(z, r, \lambda, k, l)$ :

$$
\begin{aligned}
f^{\prime \prime}(z, r, \lambda, k, l) & =\frac{\lambda(\lambda+r-1) z^{\lambda+r-2}-\lambda(\lambda-1) z^{\lambda-2}}{k-l}+\frac{r(\lambda+r-1) z^{\lambda+r-2}}{k-l} \\
& =\frac{\lambda(\lambda-1) z^{\lambda-2}}{k-l}\left(z^{r}-1\right)+\frac{r z^{\lambda+r-2}}{k-l}+\frac{r(\lambda+r-1) z^{\lambda+r-2}}{k-l} \\
& =\frac{\lambda(\lambda-1) z^{\lambda-2}}{k-l}\left(z^{r}-1\right)+\frac{r z^{\lambda+r-2}}{k-l}(2 \lambda+r-1),
\end{aligned}
$$

and by collecting some terms in brackets, we get

$$
f^{\prime \prime}(z, r, \lambda, k, l)=\frac{(\lambda-1)}{z}\left(\frac{\lambda z^{\lambda-1}}{k-l}(z-1) \phi_{r-1}(z)+\frac{r z^{\lambda+r-1}}{k-l}\right)+\frac{r z^{\lambda+r-2}}{k-l}(\lambda+r) .
$$

Now, using (24), the second derivative of the function $f(z, r, \lambda, k, l)$ will be equal to

$$
f^{\prime \prime}(z, r, \lambda, k, l)=\frac{(\lambda-1)}{z} f^{\prime}(z, r, \lambda, k, l)+\frac{r z^{\lambda+r-2}}{k-l}(\lambda+r) .
$$

Replacing $z$ by $i$ in (25) and using (18), we obtain

$$
\begin{aligned}
f^{\prime \prime}(i, r, \lambda, k, l) & =\frac{(\lambda-1)}{i} f^{\prime}(i, r, \lambda, k, l)-r i^{\lambda+r-1}+\frac{r i^{\lambda+r-2}}{k-l}(\lambda+r) \\
& =\frac{\lambda(\lambda-1)}{i^{2}} f(i, r, \lambda, k, l)+\frac{r(\lambda-1)}{i} \frac{i^{\lambda+r-1}}{k-l}+\frac{r i^{\lambda+r-2}}{k-l}(\lambda+r) \\
& =\frac{\lambda(\lambda-1)}{i^{2}} f(i, r, \lambda, k, l)+\left[\frac{r(\lambda-1)}{i} \frac{i^{\lambda+r-1}}{k-l}+\frac{r(2 \lambda+r-1)}{k-l}\right] i^{\lambda+r-2} .
\end{aligned}
$$


By iterating these above derivations for the variable $z$, and then replacing $z$ by $i$, we finally obtain

$$
\begin{aligned}
f^{(m)}(i, r, \lambda, k, l) & =\frac{\lambda(\lambda-1) \cdots(\lambda-m+1)}{i^{m}} f(i, r, \lambda, k, l)+\mathcal{A} i^{\lambda+r-m} \\
& =m !\left(\begin{array}{c}
\lambda \\
m
\end{array}\right) i^{-m} f(i, r, \lambda, k, l)+\mathcal{A} i^{\lambda+r-m}
\end{aligned}
$$

where $\mathcal{A}$ stands for some constants.

This above theory is related to the functional equations. In fact, these above progresses show that the presentation $\mathcal{P}_{M}$ in (7) can be related to the functional equations.

\section{Competing interests}

The authors declare that they have no competing interests.

\section{Authors' contributions}

All authors completed the paper together. All authors read and approved the final manuscript.

\section{Author details}

${ }^{1}$ Department of Mathematics, Faculty of Science, Selçuk University, Campus, Konya, 42075, Turkey. ${ }^{2}$ Department of Mathematics, Faculty of Arts and Science, Uludag University, Gorukle Campus, Bursa, 16059, Turkey. ${ }^{3}$ Department of Mathematics, Faculty of Art and Science, Akdeniz University, Campus, Antalya, 07058, Turkey.

\section{Acknowledgements}

All authors are partially supported by Research Project Offices of Uludağ, Selçuk and Akdeniz Universities, and TUBITAK (The Scientific and Technological Research Council of Turkey).

Received: 19 November 2012 Accepted: 9 January 2013 Published: 22 January 2013

\section{References}

1. Stillwell, J: Classical Topology and Combinatorial Group Theory. Graduate Texts in Mathematics, vol. 72 . Springer, Berlin (1980)

2. Birkhoff, GD, Lewis, D: Chromatic polynomials. Trans. Am. Math. Soc. 60, 355-451 (1946)

3. Cardoso, DM, Silva, ME, Szymanski, J: A generalization of chromatic polynomial of a graph subdivision. J. Math. Sci. 183(2), 246-254 (2012)

4. Cangul, IN, Cevik, AS, Simsek, Y: A new approach to connect algebra with analysis: relationships and applications between presentations and generating functions. Bound. Value Probl. (accepted)

5. Howie, JM: Fundamentals of Semigroup Theory. Clarendon, New York (1995)

6. Guba, V, Sapir, M: Diagram Groups. Am. Math. Soc., Providence (1997)

7. Pride, SJ: Geometric methods in combinatorial semigroup theory. In: Fountain, J (ed.) Semigroups, Formal Languages and Groups, pp. 215-232. Kluwer Academic, Dordrecht (1995)

8. Pride, SJ: Low-dimensional homotopy theory for monoids. Int. J. Algebra Comput. 5(6), 631-649 (1995)

9. Squier, CC: Word problems and a homological finiteness condition for monoids. J. Pure Appl. Algebra 49, 201-216 (1987)

10. Cevik, AS: The $p$-Cockcroft property of the semidirect products of monoids. Int. J. Algebra Comput. 13(1), 1-16 (2003)

11. Cevik, AS: Minimal but inefficient presentations of the semidirect products of some monoids. Semigroup Forum 66 , 1-17 (2003)

12. Cremanns, R, Otto, F: Finite derivation type implies the homological finiteness condition FP. J. Symb. Comput. 18, 91-112 (1994)

13. Ivanov, SV: Relation modules and relation bimodules of groups, semigroups and associative algebras. Int. J. Algebra Comput. 1, 89-114 (1991)

14. Wang, J: Finite derivation type for semi-direct products of monoids. Theor. Comput. Sci. 191(1-2), 219-228 (1998)

15. Epstein, DBA: Finite presentations of groups and 3-manifolds. Q. J. Math. 12, 205-212 (1961)

16. Saito, T: Orthodox semi-direct products and wreath products of monoids. Semigroup Forum 38, 347-354 (1989)

17. Dlab, V, Neumann, BH: Semigroups with few endomorphisms. J. Aust. Math. Soc. A 10, 162-168 (1969)

18. Ates, F, Cevik, AS: Minimal but inefficient presentations for semi-direct products of finite cyclic monoids. In: Groups St. Andrews 2005, Volume 1. London Mathematical Society Lecture Note Series, vol. 339, pp. 170-185 (2007)

19. Chang, $\mathrm{CH}, \mathrm{Ha}, \mathrm{CW}$ : A multiplication theorem for the Lerch zeta function and explicit representations of the Bernoulli and Euler polynomials. J. Math. Anal. Appl. 315, 758-767 (2006)

20. Simsek, Y: Generating functions for generalized Stirling type numbers, array type polynomials, Eulerian type polynomials and their applications. arXiv:1111.3848v2 [math.NT] 23 Nov 2011

21. Simsek, Y: Interpolation function of generalized $q$-Bernstein type polynomials and their application. In: Curves and Surfaces. Lecture Notes in Computer Science, vol. 6920, pp. 647-662. Springer, Berlin (2011)

22. Mismar, MJ, Abu-Al-Nadi, DI, Ismail, TH: Pattern synthesis with phase-only control using array polynomial technique. In: IEEE International Conference on Signal Processing and Communications (ICSPC 2007), 24-27 November 2007, Dubai, UAE (2007) 
23. Brown, KS: Cohomology of Groups. Graduate Text in Mathematics, vol. 87. Springer, Berlin (1982)

24. Agoh, T, Dilcher, K: Shortened recurrence relations for Bernoulli numbers. Discrete Math. 309, 887-898 (2009)

25. Carlitz, L: Some numbers related to the Stirling numbers of the first and second kind. Univ. Beograd Publ. Elektrotehn. Fak. Ser. Mat. Fiz. 544-576, 49-55 (1976)

26. Kim, T: $q$-Volkenborn integration. Russ. J. Math. Phys. 19, 288-299 (2002)

27. Luo, QM, Srivastava, HM: Some generalizations of the Apostol-Genocchi polynomials and the Stirling numbers of the second kind. Appl. Math. Comput. 217, 5702-5728 (2011)

28. Srivastava, HM: Some generalizations and basic (or q-) extensions of the Bernoulli, Euler and Genocchi polynomials. Appl. Math. Inf. Sci. 5, 390-444 (2011)

doi:10.1186/1687-1812-2013-15

Cite this article as: Cevik et al.: Analysis approach to finite monoids. Fixed Point Theory and Applications 2013 2013:15.

Submit your manuscript to a SpringerOpen ${ }^{\circ}$ journal and benefit from:

- Convenient online submission

- Rigorous peer review

- Immediate publication on acceptance

- Open access: articles freely available online

- High visibility within the field

- Retaining the copyright to your article

Submit your next manuscript at $>$ springeropen.com 